Article

\title{
Qualitative Analysis of Multi-Terms Fractional Order Delay Differential Equations via the Topological Degree Theory
}

\author{
Muhammad Sher ${ }^{1}$, Kamal Shah ${ }^{1}(\mathbb{D})$, Michal Fečkan ${ }^{2,3, *(\mathbb{D})}$ and Rahmat Ali Khan ${ }^{1}$ \\ 1 Department of Mathematics, University of Malakand, Dir(L), Khyber Pakhtunkhwa 18000, Pakistan; \\ msherpak55@gmail.com (M.S.); kamalshah408@gmail.com (K.S.); rahmat_alipk@yahoo.com (R.A.K.) \\ 2 Department of Mathematical Analysis and Numerical Mathematics, Faculty of Mathematics, \\ Physics and Informatics, Comenius University in Bratislava, Mlynská Dolina, 84248 Bratislava, Slovakia \\ 3 Mathematical Institute, Slovak Academy of Sciences, Štefánikova 49, 81473 Bratislava, Slovakia \\ * Correspondence: michal.feckan@fmph.uniba.sk
}

Received: 6 January 2020; Accepted: 4 February 2020; Published: 8 February 2020

\begin{abstract}
With the help of the topological degree theory in this manuscript, we develop qualitative theory for a class of multi-terms fractional order differential equations (FODEs) with proportional delay using the Caputo derivative. In the same line, we will also study various forms of Ulam stability results. To clarify our theocratical analysis, we provide three different pertinent examples.
\end{abstract}

Keywords: fractional differential equations; existence and uniqueness of solutions; topological degree method

\section{Introduction}

Fractional calculus is the fastest growing area for research in the last three decades. Nowadays it has become an important tool due to its wide range of applications in various scientific disciplines such as biology, chemistry, physics, dynamical systems, electrodynamics, etc. (see [1-6] and references therein). Its importance can also be explored in other fields like fluid dynamics traffic models, oscillation due to earthquakes, flow in porous media due to seepage, etc.

Therefore such problems have been considered from different aspects to check whether the problem of a differential or integral equation that is to be investigated has a solution or not. To guarantee the answer, the existence theory is used to find the conditions under which the problem under investigation has a solution or not. Therefore, existence and uniqueness are the important aspects of differential equations (DEs), which have been studied very openly by different authors using various approaches (for example, see [7,8], etc.). Classical fixed point theory has been utilized to study existence and uniqueness for certain problems $[9,10]$. Using these results, one needs to establish strong compact conditions, which shorten the study to some boundary value problems (BVPs). To manage this limitation and to generalize the techniques to greater extent for BVPs, researchers have been looking for a tool of nonlinear analysis. One of the important tools is topological degree theory which needs weak compact conditions instead of strong compact conditions for operation. The suggested method provides very basic criteria for existence results for many problems. Enormous numbers of problems, both linear and nonlinear DEs and FODEs, have been investigated for existence results by researchers. Mawhin [11] applied degree theory to develop appropriate results for the given BVPs to derive existence and uniqueness results:

$$
\begin{aligned}
& \mathrm{w}^{\prime \prime}(\mathrm{t})+\mathfrak{f}\left(\mathrm{t}, \mathrm{w}(\mathrm{t}), \mathrm{w}^{\prime}(\mathrm{t})\right)=0, \quad \mathrm{t} \in[0, \pi] \\
& \mathrm{w}(0)=\mathrm{w}(\pi)=0
\end{aligned}
$$


and

$$
\begin{aligned}
& \mathrm{w}^{\prime \prime}(\mathrm{t})+\mathfrak{f}(\mathrm{t}, \mathrm{w}(\mathrm{t}))=0, \quad \mathrm{t} \in[0,1] \\
& \mathrm{w}(0)=\mathrm{w}(1)=0 .
\end{aligned}
$$

Isaia [12] has applied the method of degree theory to form some adequate results about existence and uniqueness results to the integral equations given by

$$
\mathrm{w}(\mathrm{t})=\varphi(\mathrm{t}, \mathrm{w}(\mathrm{t}))+\int_{\mathfrak{a}}^{\mathfrak{b}} \psi(\mathrm{t}, \mathrm{s}, \mathrm{w}(\mathrm{s})) \mathrm{ds}, \quad \mathrm{t} \in \mathrm{J}=[a, b],
$$

where $\varphi \in \mathrm{C}[\mathrm{J} \times \mathrm{R}, \mathrm{R}], \psi \in \mathrm{C}[\mathrm{J} \times \mathrm{J} \times \mathrm{R}, \mathrm{R}]$ under some growth conditions.

One of the weighty classes of DEs is known as pantograph equations (PEs), which involve proportional type delay. In the 1960s, the British railways wanted to make the electric locomotive faster. An important construction was the pantograph, which collects current from an overhead wire. Therefore, Ockendon and Tayler studied the motion of the pantograph for an electric locomotive [13]. The above mentioned class of DEs has a large numbers of applications in real-world scientific disciplines such as dynamical systems, quantum mechanics and electrodynamics. Particularly, as mentioned above, the mentioned delay DEs are used to collect current from overhead wire. Therefore several researchers have attempted to develop conditions for existence and uniqueness of solution to the aforesaid DEs. Many authors also have considered delay DEs using analytical and numerical techniques [14-16].

Liu and Li [16] studied the following multi-term FODEs with proportional delay for existence and uniqueness via fixed point theory

$$
\left\{\begin{array}{l}
{ }^{c} D_{0+}^{\kappa} w(t)=f\left(t, w(t), w\left(\gamma_{1} t\right), \ldots, w\left(\gamma_{n} t\right)\right), \quad t \in J=[0, \theta], \\
w(0)=w_{0},
\end{array}\right.
$$

where $0<\kappa \leq 1$, for $i=1,2, \ldots, \mathrm{n}, \lambda_{i} \in(0,1)$ and $\mathfrak{f} \in \mathrm{C}\left[\mathrm{J} \times \mathrm{R}^{\mathrm{m}}, \mathrm{R}\right]$.

Since most nonlinear problems cannot be solved for exact solutions, we need powerful numerical or analytical techniques. For good numerical results one needs stable algorithms and methods. For such needs, the stability theory was founded. This aspect is important in numerical study and optimization procedures. In the literature, there are different type of stability such as exponential, Mittag-Leffler and Lyapunov type. These stabilities were studied for DEs of ordinary order. In the past few years stability results have been generalized for linear and nonlinear FODEs, (for details, see $[17,18]$ ). To establish these stabilities for DEs, some of them need a pre-defined Lyapunov function which is sometimes very difficult and also needs much time. On the other hand the exponential and Mittag-Leffler stability involving exponential functions have difficulties during numerical analysis of the problems. To handle these difficulties Ulam [19] in 1940 introduced another kind of stability, known as Ulam-Hyer's (UH) stability which was further studied by Hyer [20] in 1941. For the first time Wang [21] studied the UH stability for the impulsive ordinary DEs in 2012. UH stability for DEs of different orders have been studied by different authors (see [22,23]). Further we state that the stability analysis is one of the basic problems in the fields of systems and signal processing and control.

Since then, the evolution of a physical system in time has been described using initial value problems. However, this is less informative. Therefore, to get more and better information, the initial (local) conditions are replaced nonlocal conditions. In fact, nonlocal conditions give a better effect as compared to local initial conditions and also the measurement due to nonlocal conditions is usually more precise than the one measurement produced by local conditions. Therefore investigation of problems under nonlocal initial or boundary conditions is one of the important areas of research in recent times (for detail we refer to $[24,25]$ ). Inspired by the aforesaid work, this research aims to study Equation (5) under generalized nonlocal integral boundary condition as: 


$$
\left\{\begin{array}{l}
{ }^{c} \mathrm{D}_{0+}^{\kappa} \mathrm{w}(\mathrm{t})=\mathfrak{f}\left(\mathrm{t}, \mathrm{w}(\mathrm{t}), \mathrm{w}\left(\gamma_{1} \mathrm{t}\right), \ldots, \mathrm{w}\left(\gamma_{\mathrm{n}} \mathrm{t}\right)\right), \quad \mathrm{t} \in \mathrm{J} \\
\mathrm{w}(0)-\mathrm{w}_{0}=\int_{0}^{\theta} \frac{(\theta-\mathrm{s})^{\kappa-1}}{\Gamma(\kappa)} \mathfrak{g}(\mathrm{s}, \mathrm{w}(\mathrm{s})) \mathrm{ds}
\end{array}\right.
$$

where $0<\kappa \leq 1$, for $i=1,2, \ldots, \mathrm{n}, \lambda_{i} \in(0,1)$ and $\mathfrak{g} \in \mathrm{C}[\mathrm{J} \times \mathrm{R}, \mathrm{R}], \mathfrak{f} \in \mathrm{C}\left[\mathrm{J} \times \mathrm{R}^{\mathrm{m}}, \mathrm{R}\right]$, for existence and uniqueness of solutions using the mentioned method. Moreover, some adequate results of various UH type stabilities such as UH stability, generalized UH (GUH) stability, UH-Rasaias (UHR) stability and generalized UHR (GUHR) stability are established. Finally the analysis is justified by some examples.

\section{Fundamental Material}

Here, we provide some fundamental material about fractional calculus, topological degree theory and UH type stability.

Definition 1 ([10]). If $\kappa \in \mathrm{R}_{+}$, then integral of fractional order for the function $\mathrm{w} \in \mathrm{L}^{1}(\mathrm{~J}, \mathrm{R})$ is expressed as

$$
\mathrm{I}_{0+}^{\kappa} \mathrm{W}(\mathrm{t})=\frac{1}{\Gamma(\kappa)} \int_{0}^{\mathrm{t}}(\mathrm{t}-\mathrm{s})^{\kappa-1} \mathrm{~W}(\mathrm{~s}) \mathrm{ds} .
$$

Definition 2 ([10]). THe derivative of fractional order to a function $\mathrm{w}$ on the interval $\mathrm{J}$ in Caputo sense is expressed as

$$
{ }^{c} \mathrm{D}_{0+}^{\kappa} \mathrm{w}(\mathrm{t})=\frac{1}{\Gamma(\mathrm{r}-\kappa)} \int_{0}^{\mathrm{t}}(\mathrm{t}-\mathrm{s})^{\mathrm{r}-\kappa-1} \mathrm{w}^{(\mathrm{r})}(\mathrm{s}) \mathrm{ds},
$$

where $\mathrm{r}=[\kappa]+1$ and $[\kappa]$ represents an integral part of $\kappa$.

Lemma 1 ([10]). The solution of FODE

$$
{ }^{c} \mathrm{D}_{0+}^{\kappa} \mathrm{w}(\mathrm{t})=0, \quad \mathrm{r}-1<\kappa \leq \mathrm{r},
$$

is given as $\mathrm{w}(\mathrm{t})=\sum_{i=0}^{\mathrm{r}-1} \mathrm{c}_{i} \mathrm{t}^{i}$, where $\mathrm{c}_{i} \in \mathrm{R}, i=0,1,2, \ldots, \mathrm{r}-1$.

Lemma 2 ([10]). Moreover, for the given FODE ${ }^{c} \mathrm{D}_{0+}^{\kappa} \mathrm{w}(\mathrm{t})=\mathfrak{y}(\mathrm{t})$

$$
\mathrm{w}(\mathrm{t})=\mathrm{I}_{+0}^{\kappa}[\mathfrak{y}(\mathrm{t})]+\sum_{i=0}^{\mathrm{r}-1} \mathrm{c}_{i} \mathrm{t}^{i}
$$

results holds, for $\mathrm{c}_{i} \in \mathrm{R}, i=0,1,2, \ldots, \mathrm{r}-1$.

In the following $\mathcal{Y}=\mathrm{C}[\mathrm{J}, \mathrm{R}]$ will be Banach space with norm $\|\mathrm{w}\|=\sup \{|\mathrm{w}(\mathrm{t})|, \mathrm{t} \in \mathrm{J}\}$ and the family $\mathbf{W} \subset \mathcal{P}(\mathcal{Y})$ represents all its bounded sets. Below are some notions and results recalled from [12].

Definition 3. The mapping $\chi: \mathbf{W} \rightarrow \mathrm{R}_{+}$for Kuratowski measure of non-compactness is defined as

$$
\chi(\mathcal{W})=\inf \{\epsilon>0\},
$$

where $\mathcal{W} \in \mathbf{W}$ is covered by finite sets with diameter $\leq \epsilon$

Proposition 1. The mapping $\chi$ due to non-compactness enjoys the properties given below:

(i) $\chi(\mathcal{W})=0 \Longleftrightarrow \mathcal{W}$ is compact relatively.

(ii) $\chi$ is a seminorm, i.e., $\chi(\sigma \mathcal{W})=|\sigma| \chi(\mathcal{W}), \sigma \in \mathrm{R}$ and $\chi\left(\mathcal{W}_{1}+\mathcal{W}_{2}\right) \leq \chi\left(\mathcal{W}_{1}\right)+\chi\left(\mathcal{W}_{2}\right)$.

(iii) $\mathcal{W}_{1} \subset \mathcal{W}_{2}$ implies $\chi\left(\mathcal{W}_{1}\right) \leq \chi\left(\mathcal{W}_{2}\right) ; \chi\left(\mathcal{W}_{1} \cup \mathcal{W}_{2}\right)=\max \left\{\chi\left(\mathcal{W}_{1}\right), \chi\left(\mathcal{W}_{2}\right)\right\}$. 
(iv) $\chi(\operatorname{conv\mathcal {W}})=\chi(\mathcal{W})$.

(v) $\chi(\mathcal{W})=\chi(\mathcal{W})$

Definition 4. Let the map $\Lambda: \Phi \rightarrow \mathcal{Y}$ be bounded and continuous, where $\Phi \subset \mathcal{Y}$. Then $\Lambda$ is $\chi$-Lipschitz if $\exists$ $\mathcal{K} \geq 0$ such that

$$
\chi(\Lambda(\mathcal{W})) \leq \mathcal{K} \chi(\mathcal{W}), \forall \quad \mathcal{W} \subset \Phi \quad \text { bounded }
$$

Further, if $\mathcal{K}<1$ then $\Lambda$ is strict $\chi$-contraction.

Proposition 2. Let $\Lambda, \Pi: \Phi \rightarrow \mathcal{Y}$ be $\chi$-Lipschitz for constants $\mathcal{K}$ and $\mathcal{K}^{\prime}$, respectively, and $\Lambda+\Pi: \Phi \rightarrow \mathcal{Y}$ also be $\chi$-Lipschitz for constants $\mathcal{K}+\mathcal{K}^{\prime}$.

Proposition 3. $\Lambda$ is $\chi$-Lipschitz for constant $\mathcal{K}=0$ If $\Lambda: \Phi \rightarrow \mathcal{Y}$ is compact.

Proposition 4. If $\Lambda: \Phi \rightarrow \mathcal{Y}$ is Lipschitz for constant $\mathcal{K}$, then $\Lambda$ is $\chi$-Lipschitz for the same constant $\mathcal{K}$.

Theorem 1 ([12]). Let $\Lambda: \mathcal{Y} \rightarrow \mathcal{Y}$ be $\chi$-condensing and

$$
\Theta=\{\omega \in \mathcal{Y}: \exists \quad \mu \in[0,1] \quad \ni \quad \omega=\mu \Lambda\} .
$$

If $\Theta$ is a bounded set in $\mathcal{Y}$, then $\exists \mathrm{r}>0 \ni \Theta \subset \mathcal{W}_{\mathrm{r}}(0)$, then the degree

$$
\operatorname{deg}\left(I-\mu \Lambda, \mathcal{W}_{\mathrm{r}}(0), 0\right)=1, \quad \forall \quad \mu \in[0,1] .
$$

Consequently, $\Lambda$ has at least one fixed point and the set of the fixed points of $\Lambda$ lies in $\mathcal{W}_{\mathrm{r}}(0)$.

The following definitions are recalled from [26].

Definition 5. The Equation (5) is $U H$ stable if for every $\epsilon>0, \exists \mathcal{C}_{\mathrm{q}} \in \mathrm{R}^{+}$and $\mathrm{w} \in \mathcal{Y}$ is any solution of

$$
\left|{ }^{\mathrm{c}} \mathrm{D}_{0+}^{\kappa} \mathrm{w}(\mathrm{t})-\mathfrak{f}\left(\mathrm{t}, \mathrm{w}(\mathrm{t}), \mathrm{w}\left(\gamma_{1} \mathrm{t}\right), \ldots, \mathrm{w}\left(\gamma_{\mathrm{n}} \mathrm{t}\right)\right)\right| \leq \epsilon, \quad \forall \mathrm{t} \in \mathrm{J}=[0, \theta],
$$

$\exists$ a unique solution $\overline{\mathrm{w}} \in \mathcal{Y}$ of (5), such that

$$
|\mathrm{w}-\overline{\mathrm{w}}| \leq \mathcal{C}_{\mathrm{q}} \epsilon, \quad \forall \mathrm{t} \in \mathrm{J} .
$$

Definition 6. The Equation (5) is GUH stable if $\exists \xi \in C\left(R^{+}, R^{+}\right), \xi(0)=0$, such that for any solution $\mathrm{w} \in \mathcal{Y}$ of (13) there is a unique solution $\overline{\mathrm{w}} \in \mathcal{Y}$ of (5), such that

$$
|\mathrm{w}-\overline{\mathrm{w}}| \leq \xi(\epsilon), \quad \forall \mathrm{t} \in \mathrm{J} .
$$

Definition 7. The Equation (5) is UHR stable with respect to $\zeta \in \mathrm{C}\left[\mathrm{J}, \mathrm{R}^{+}\right]$, if $\exists \mathcal{C}_{q} \in \mathrm{R}^{+}$such that for every $\epsilon>0$ and for any solution $\mathrm{w} \in \mathcal{Y}$ of the inequality

$$
\left|{ }^{c} \mathrm{D}_{0+}^{\kappa} \mathrm{w}(\mathrm{t})-\mathfrak{f}\left(\mathrm{t}, \mathrm{w}(\mathrm{t}), \mathrm{w}\left(\gamma_{1} \mathrm{t}\right), \cdots, \mathrm{w}\left(\gamma_{\mathrm{n}} \mathrm{t}\right)\right)\right| \leq \zeta(\mathrm{t}) \epsilon, \quad \forall \mathrm{t} \in \mathrm{J},
$$

there is a unique solution $\overline{\mathrm{w}} \in \mathcal{Y}$ of (5), such that

$$
|\mathrm{w}-\overline{\mathrm{w}}| \leq \mathcal{C}_{q} \epsilon \zeta(\mathrm{t}), \quad \forall \mathrm{t} \in \mathrm{J} .
$$

Definition 8. The Equation (5) is GUHR stable with respect to $\zeta \in \mathrm{C}\left[\mathrm{J}, \mathrm{R}^{+}\right]$if $\exists \mathcal{C}_{q, \zeta} \in \mathrm{R}^{+}$such that for every $\epsilon>0$ and for any solution $\mathrm{w} \in \mathcal{Y}$ of the inequality (14) there is a unique solution $\overline{\mathrm{w}} \in \mathcal{Y}$ of (5), such that

$$
|\mathrm{w}-\overline{\mathrm{w}}| \leq \mathcal{C}_{q, \zeta} \zeta(\mathrm{t}), \quad \forall \mathrm{t} \in \mathrm{J} .
$$


Remark 1. A function $\overline{\mathrm{w}} \in \mathcal{Y}$ is a solution of (13) if there is a function $\eta(\mathrm{t}) \in \mathrm{C}(\mathrm{J} ; \mathrm{R})$ (dependent on $\overline{\mathrm{w}})$, such that

(i) $|\eta(\mathrm{t})| \leq \epsilon, \quad \forall \quad \mathrm{t} \in \mathrm{J}$.

(ii) ${ }^{c} \mathrm{D}^{\kappa} \overline{\mathrm{w}}(\mathrm{t})=\mathfrak{f}\left(\mathrm{t}, \overline{\mathrm{w}}(\mathrm{t}), \overline{\mathrm{w}}\left(\gamma_{1} \mathrm{t}\right), \ldots, \overline{\mathrm{w}}\left(\gamma_{\mathrm{n}} \mathrm{t}\right)\right)+\eta(\mathrm{t}), \quad \forall \mathrm{t} \in \mathrm{J}$.

Remark 2. A function $\overline{\mathrm{w}} \in \mathcal{Y}$ is a solution of (14) if there is a function $\eta(\mathrm{t}) \in \mathrm{C}(\mathrm{J} ; \mathrm{R})$ (dependent on $\overline{\mathrm{w}})$, such that

(i) $|\eta(\mathrm{t})| \leq \epsilon \varphi, \quad \forall \quad \mathrm{t} \in \mathrm{J}$.

(ii) ${ }^{\mathrm{c}} \mathrm{D}^{\kappa} \overline{\mathrm{w}}(\mathrm{t})=\mathfrak{f}\left(\mathrm{t}, \overline{\mathrm{w}}(\mathrm{t}), \overline{\mathrm{w}}\left(\gamma_{1} \mathrm{t}\right), \cdots, \overline{\mathrm{w}}\left(\gamma_{\mathrm{n}} \mathrm{t}\right)\right)+\eta(\mathrm{t}), \quad \forall \mathrm{t} \in \mathrm{J}$.

\section{Main Results}

In this section we study existence results for nonlinear delay FODEs under integral boundary condition, we use $\mathrm{J}=[0, \theta]$ and $\mathfrak{Z}=\mathrm{C}(\mathrm{J})$.

By Lemma 2, the considered problem (5) is converted to the following delay integral equation as

$$
\mathrm{w}(\mathrm{t})=\mathrm{w}_{0}+\int_{0}^{\theta} \frac{(\theta-\mathrm{s})^{\kappa-1}}{\Gamma(\kappa)} \mathfrak{g}(\mathrm{s}, \mathrm{w}(\mathrm{s})) \mathrm{ds}+\int_{0}^{\mathrm{t}} \frac{(\mathrm{t}-\mathrm{s})^{\kappa-1}}{\Gamma(\kappa)} \mathfrak{f}\left(\mathrm{s}, \mathrm{w}(\mathrm{s}), \mathrm{w}\left(\gamma_{1} \mathrm{~s}\right), \cdots, \mathrm{w}\left(\gamma_{\mathrm{n}} \mathrm{s}\right)\right) \mathrm{ds},
$$

for $\mathrm{t} \in \mathrm{J}$ and $\mathfrak{g} \in \mathrm{C}[\mathrm{J} \times \mathrm{R}, \mathrm{R}], \mathfrak{f} \in \mathrm{C}\left[\mathrm{J} \times \mathrm{R}^{\mathrm{m}}, \mathrm{R}\right]$, such that

(i) There exist $\mathfrak{a}, \mathfrak{b} \geq 0, \mathfrak{c}_{1} \in[0,1)$ such that

$$
\|\mathfrak{g}(\mathrm{t}, \mathrm{w})\| \leq \mathfrak{a}\|\mathrm{w}\|^{\mathfrak{c}_{1}}+\mathfrak{b}
$$

$\forall(\mathrm{t}, \mathrm{w}) \in \mathrm{J} \times \mathrm{R}$.

(ii) There exist $\mathcal{K}_{1} \in[0,1)$ such that

$$
\left\|\mathfrak{g}\left(\mathrm{t}, \mathrm{w}_{1}\right)-\mathfrak{g}\left(\mathrm{t}, \mathrm{w}_{2}\right)\right\| \leq \mathcal{K}_{1}\left\|\mathrm{w}_{1}-\mathrm{w}_{2}\right\|
$$

for every $\left(t, w_{1}\right),\left(t, w_{2}\right) \in J \times R$.

(iii) There exist $\mathfrak{c}, \mathfrak{d}$ and $\mathfrak{c}_{\mathfrak{f}} \geq 0, \mathfrak{c}_{2} \in[0,1)$, such that

$$
\begin{aligned}
\left|\mathfrak{f}\left(\mathrm{t}, \mathrm{w}(\mathrm{t}), \mathrm{w}\left(\gamma_{1} \mathrm{t}\right), \cdots, \mathrm{w}\left(\gamma_{\mathrm{n}} \mathrm{t}\right)\right)\right| & \leq \mathfrak{c}\left[|\mathrm{w}(\mathrm{t})|^{\mathfrak{c}_{2}}+\left|\mathrm{w}\left(\gamma_{1} \mathrm{t}\right)\right|^{\mathfrak{c}_{2}}, \cdots,\left|\mathrm{w}\left(\gamma_{\mathrm{n}} \mathrm{t}\right)\right|^{\mathfrak{c}_{2}}\right]+\mathfrak{d} \\
& \leq \mathfrak{c}_{f}|\mathrm{w}(\mathrm{t})|^{\mathfrak{c}_{2}}+\mathfrak{d}, \text { where } \mathfrak{c}_{\mathfrak{f}}=(\mathrm{n}+1) \mathfrak{c}
\end{aligned}
$$

for all $\left(t, w(t), w\left(\gamma_{1} t\right), \cdots, w\left(\gamma_{n} t\right)\right) \in J \times R^{m}$.

The above three conditions will be used to show the existence and uniqueness of the solution of (5).

Here we define some operators as

$$
\begin{aligned}
\mathcal{A}: \mathfrak{Z} \rightarrow \mathfrak{Z},(\mathcal{A w})(\mathrm{t}) & =\mathrm{w}_{0}+\int_{0}^{\theta} \frac{(\theta-\mathrm{s})^{\mathcal{K}-1}}{\Gamma(\kappa)} \mathfrak{g}(\mathrm{s}, \mathrm{w}(\mathrm{s})) \mathrm{ds} \\
\mathcal{B}: \mathfrak{Z} \rightarrow \mathfrak{Z},(\mathcal{B} \mathrm{w})(\mathrm{t}) & =\int_{0}^{\mathrm{t}} \frac{(\mathrm{t}-\mathrm{s})^{\mathcal{k}-1}}{\Gamma(\mathcal{K})} \mathfrak{f}\left(\mathrm{s}, \mathrm{w}(\mathrm{s}), \mathrm{w}\left(\gamma_{1} \mathrm{~s}\right), \cdots, \mathrm{w}\left(\gamma_{\mathrm{n}} \mathrm{s}\right)\right) \mathrm{ds}, \\
\mathcal{M}: \mathfrak{Z} \rightarrow \mathfrak{Z}, \mathcal{M w} & =\mathcal{A w}+\mathcal{B} \mathrm{w} .
\end{aligned}
$$

Then (15) in operator form becomes

$$
\mathrm{w}=\mathcal{M} \mathrm{w}
$$

The fixed point of $\mathcal{M}$ will insure the existence of the solution of (5). 
Proposition 5. The map $\mathcal{A}: \mathfrak{Z} \rightarrow \mathfrak{Z}$ is Lipschitz with constant $\mathcal{L}$. Consequently it is $\chi$-Lipchitz with constant $\mathcal{L}$.

Proof. Consider

$$
\begin{aligned}
\|\mathcal{A} \mathrm{u}-\mathcal{A} \mathrm{w}\| & =\sup \{|(\mathcal{A u})(\mathrm{t})-(\mathcal{A w})(\mathrm{t})|, \mathrm{t} \in \mathrm{J}\} \\
& \leq \sup \left\{\int_{0}^{\theta}\left|\frac{(\theta-\mathrm{s})^{\kappa-1}}{\Gamma(\kappa)}\right||\mathfrak{g}(\mathrm{s}, \mathrm{u}(\mathrm{s}))-\mathfrak{g}(\mathrm{s}, \mathrm{w}(\mathrm{s}))| \mathrm{ds}, \mathrm{s} \in \mathrm{J}\right\} \\
& \leq \mathcal{K}_{1} \sup \left\{\int_{0}^{\theta}\left|\frac{(\theta-\mathrm{s})^{\kappa-1}}{\Gamma(\kappa)}\right||\mathrm{u}(\mathrm{s})-\mathrm{w}(\mathrm{s})| \mathrm{ds}, \mathrm{s} \in \mathrm{J}\right\} \\
& =\mathcal{L}\|\mathrm{u}-\mathrm{w}\|, \text { where } \mathcal{L}=\frac{\mathcal{K}_{1} \theta^{\kappa}}{\Gamma(\kappa+1)} \text { and } \mathcal{K}_{1} \text { be such that } \mathcal{L}<1 .
\end{aligned}
$$

for every $\mathrm{u}, \mathrm{w} \in \mathfrak{Z}$. Thus $\mathcal{A}$ is $\chi$-Lipschitz with constant $\mathcal{L}$, proposition (4).

Using condition $(i), \mathcal{A}$ obeys the result given by:

$$
\|\mathcal{A} w\| \leq \mathfrak{a}^{\prime}\|\mathrm{w}\|^{\mathfrak{c}_{1}}+\mathfrak{b}^{\prime}, \text { where } \mathfrak{a}^{\prime}=\frac{\mathfrak{a} \theta^{\kappa}}{\Gamma(\kappa+1)} \text { and } \mathfrak{b}^{\prime}=\left|\mathrm{w}_{0}\right|+\frac{\mathfrak{b} \theta^{\kappa}}{\Gamma(\kappa+1)},
$$

for every $\mathrm{w} \in \mathfrak{Z}$.

Proposition 6. The map $\mathcal{B}: \mathfrak{Z} \rightarrow \mathfrak{Z}$ is compact. Consequently $\mathcal{B}$ is $\chi$-Lipschitz with zero constant.

Proof. To prove $\mathcal{B}$ is a continuous, let $\left\{\mathrm{w}_{\mathrm{n}}\right\} \subset \mathfrak{Z}, \mathrm{w} \in \mathfrak{Z}$ be such that $\left\|\mathrm{w}_{\mathrm{n}}-\mathrm{w}\right\| \rightarrow 0$ as $\mathrm{n} \rightarrow \infty$. We must have to show $\left\|\mathcal{B}_{n}-\mathcal{B w}\right\| \rightarrow 0$ as $\mathrm{n} \rightarrow \infty$. For $\epsilon>0, \exists \mathcal{K} \geq 0$ such that

$$
\begin{aligned}
\left\|\mathrm{w}_{\mathrm{n}}\right\| & \leq \mathcal{K}, \forall \mathrm{n} \in \mathrm{N}, \\
\|\mathrm{w}\| & \leq \mathcal{K} .
\end{aligned}
$$

Since $\mathfrak{f}$ is continuous, $\mathfrak{f}$ is uniform continuous on $\mathfrak{Z} \times \overline{\mathcal{S}}(0, \mathcal{K})$, where $\overline{\mathcal{S}}(0, \mathcal{K})=$ $\left\{\mathrm{q} \in \mathrm{R}^{m}: \mathrm{d}(\mathrm{q}, 0) \leq \mathcal{K}\right\}$ and $\mathrm{d}$ is usual metric on $\mathrm{R}^{\mathrm{m}}$. Using the definition of uniform continuity $\exists \delta=\delta(\epsilon)$ such that

$$
\left|\mathfrak{f}\left(\mathfrak{t}_{1}, \rho, \rho_{1}, \rho_{2}, \cdots, \rho_{\mathrm{n}}\right)-\mathfrak{f}\left(\mathrm{t}_{2}, \sigma, \sigma_{1}, \sigma_{2}, \cdots, \sigma_{\mathrm{n}}\right)\right|<\epsilon^{\prime}, \text { where } \epsilon^{\prime}=\frac{\epsilon \Gamma(\kappa+1)}{\theta^{\kappa}},
$$

for every $\left(\mathrm{t}_{1}, \rho, \rho_{1}, \rho_{2}, \cdots, \rho_{\mathrm{n}}\right),\left(\mathrm{t}_{2}, \sigma, \sigma_{1}, \sigma_{2}, \cdots, \sigma_{\mathrm{n}}\right) \in \mathrm{J} \times \overline{\mathrm{S}}(0, \mathcal{K})$ such that $\left|\mathrm{t}_{1}-\mathrm{t}_{2}\right|+|\rho-\sigma|+\mid \rho_{1}-$ $\sigma_{1}|+| \rho_{2}-\sigma_{2}|+\cdots+| \rho_{\mathrm{n}}-\sigma_{\mathrm{n}} \mid<\delta$. Since $\left\|\mathrm{w}_{\mathrm{n}}-\mathrm{w}\right\| \rightarrow 0$ as $\mathrm{n} \rightarrow \infty$ so for $\epsilon>0 \exists \mathrm{m} \in \mathrm{N}$ such that $\sup \left|\mathrm{w}_{\mathrm{n}}(\mathrm{t})-\mathrm{w}(\mathrm{t})\right|<\epsilon$, for every $\mathrm{n} \geq \mathrm{m}$. Thus

$$
\begin{aligned}
\left\|\mathcal{B} \mathrm{w}_{\mathrm{n}}-\mathcal{B} \mathrm{w}\right\| & =\sup _{\mathrm{t} \in \mathrm{J}} \mid \int_{0}^{\mathrm{t}} \frac{(\mathrm{t}-\mathrm{s})^{\kappa-1}}{\Gamma(\kappa)} \mathfrak{f}\left(\mathrm{s}, \mathrm{w}_{\mathrm{n}}(\mathrm{s}), \mathrm{w}_{\mathrm{n}}\left(\gamma_{1} \mathrm{~s}\right), \cdots, \mathrm{w}\left(\gamma_{\mathrm{n}} \mathrm{s}\right)\right) \mathrm{ds} \\
& -\int_{0}^{\mathrm{t}} \frac{(\mathrm{t}-\mathrm{s})^{\kappa-1}}{\Gamma(\kappa)} \mathfrak{f}\left(\mathrm{s}, \mathrm{w}(\mathrm{s}), \mathrm{w}\left(\gamma_{1} \mathrm{~s}\right), \cdots, \mathrm{w}\left(\gamma_{\mathrm{n}} \mathrm{s}\right)\right) \mathrm{ds} \mid \\
& \leq \sup _{\mathrm{t} \in \mathrm{J}} \int_{0}^{\mathrm{t}}\left|\frac{(\mathrm{t}-\mathrm{s})^{\kappa-1}}{\Gamma(\kappa)}\right| \mid \mathfrak{f}\left(\mathrm{s}, \mathrm{w}_{\mathrm{n}}(\mathrm{s}), \mathrm{w}_{\mathrm{n}}\left(\gamma_{1} \mathrm{~s}\right), \cdots, \mathrm{w}\left(\gamma_{\mathrm{n}} \mathrm{s}\right)\right) \\
& -\mathfrak{f}\left(\mathrm{s}, \mathrm{w}(\mathrm{s}), \mathrm{w}\left(\gamma_{1} \mathrm{~s}\right), \cdots, \mathrm{w}\left(\gamma_{\mathrm{n}} \mathrm{s}\right)\right) \mid \mathrm{ds} \\
& \leq \frac{\epsilon^{\prime} \theta^{\kappa}}{\Gamma(\kappa+1)} \\
& <\epsilon .
\end{aligned}
$$


To prove $\mathcal{B}$ is compact, consider a bounded set $\mathcal{S} \subset \mathfrak{Z}$. Let $\mathcal{K} \geq 0$, such that

$$
\|\mathrm{w}\| \leq \mathcal{K}
$$

for every $\mathrm{w} \in \mathcal{S}$. $\mathcal{B}$ satisfies the growth condition as

$$
\|\mathcal{B} w\| \leq \mathfrak{c}^{\prime}\|\mathrm{w}\| \mathbb{c}^{2}+\mathfrak{d}^{\prime}, \text { where } \mathfrak{c}^{\prime}=\frac{\mathfrak{c}_{\mathfrak{f}} \theta^{\kappa}}{\Gamma(\kappa+1)} \text { and } \mathfrak{d}^{\prime}=\frac{\mathfrak{d} \theta^{\kappa}}{\Gamma(\kappa+1)^{\prime}}
$$

for every $\mathrm{w} \in \mathcal{S}$. Now using the above growth condition, we have

$$
\|\mathcal{B} w\| \leq \mathfrak{c}^{\prime} \mathcal{K}^{\mathfrak{c}_{2}}+\mathfrak{d}^{\prime}
$$

So $\mathcal{B}(\mathcal{S})$ is bounded in $\mathfrak{Z}$. Since $\mathfrak{f} \in \mathrm{C}\left[\mathrm{J} \times \mathrm{R}^{\mathrm{m}}, \mathrm{R}\right]$. Thus $\mathfrak{f}$ is uniform continuous on $\mathrm{J} \times \overline{\mathcal{S}}(0, \mathcal{K})$, let $\mathrm{t}_{1}, \mathrm{t}_{2} \in[0, \theta]$ such that $\mathrm{t}_{1} \geq \mathrm{t}_{2} \mid$, then

$$
\begin{aligned}
\left|(\mathcal{B} \mathrm{w})\left(\mathrm{t}_{1}\right)-(\mathcal{B} \mathrm{w})\left(\mathrm{t}_{2}\right)\right| & \leq\left[\int_{0}^{\mathrm{t}_{1}} \frac{(\mathrm{t}-1-\mathrm{s})^{\kappa-1}}{\Gamma(\kappa)}-\int_{0}^{\mathrm{t}_{2}} \frac{\left(\mathrm{t}_{2}-\mathrm{s}\right)^{\kappa-1}}{\Gamma(\kappa)}\right]\left|\mathfrak{f}\left(\mathrm{s}, \mathrm{w}(\mathrm{s}), \mathrm{w}\left(\gamma_{1} \mathrm{~s}\right), \cdots, \mathrm{w}\left(\gamma_{\mathrm{n}} \mathrm{s}\right)\right)\right| \mathrm{ds} \\
& \leq \frac{\left[\mathfrak{c}_{f} \mathcal{K}^{\mathfrak{c}_{2}}+\mathfrak{d}\right]}{\Gamma(\kappa+1)}\left(\mathrm{t}_{1}^{\kappa}-\mathrm{t}_{2}^{\kappa}\right) .
\end{aligned}
$$

The right side of (18) tends to zero when $t_{1} \rightarrow t_{2}$. Therefore, $\left\|(\mathcal{B} w)\left(t_{1}\right)-(\mathcal{B} w)\left(t_{2}\right)\right\| \rightarrow 0$ on using $t_{1} \rightarrow t_{2}$. Hence in light of the Arzelá-Ascoli theorem, $\mathcal{B}(\mathcal{S}) \subset \mathfrak{Z}$ is relatively compact. Thus $\mathcal{B}$ is $\chi$-Lipschitz with zero constant.

\section{Existence Criteria}

In this part of our paper we derive results for the existence and uniqueness of the solution to the considered problem.

Theorem 2. If the functions $\mathfrak{g}$ and $\mathfrak{f}$ satisfy conditions (i), (ii) and (iii), then the integral equation

$$
\mathrm{w}(\mathrm{t})=\mathrm{w}_{0}+\int_{0}^{\theta} \frac{(\theta-\mathrm{s})^{\kappa-1}}{\Gamma(\kappa)} \mathfrak{g}(\mathrm{s}, \mathrm{w}(\mathrm{s})) \mathrm{ds}+\int_{0}^{\mathrm{t}} \frac{(\mathrm{t}-\mathrm{s})^{\kappa-1}}{\Gamma(\kappa)} \mathfrak{f}\left(\mathrm{s}, \mathrm{w}(\mathrm{s}), \mathrm{w}\left(\gamma_{1} \mathrm{~s}\right), \cdots, \mathrm{w}\left(\gamma_{\mathrm{n}} \mathrm{s}\right)\right) \mathrm{ds}
$$

possesses at least one solution, $\mathrm{w} \in \mathfrak{Z}$, and the solution(s) set is bounded in $\mathfrak{Z}$.

Proof. The operators $\mathcal{A}, \mathcal{B}, \mathcal{M}: \mathfrak{Z} \rightarrow \mathfrak{Z}$ are bounded and continuous. Moreover, $\mathcal{A}$ is $\chi$-Lipschitz with constant $\mathcal{L} \in[0,1)$ and $\mathcal{B}$ is $\chi$-Lipschitz with zero constant (Propositions 4 and 3 ). $\mathcal{M}$ is a strict $\chi$-contraction with constant $\mathcal{L}$ (Proposition 2).

Consider the set

$$
\Theta=\{\mathrm{w} \in \mathfrak{Z}: \exists \mu \in[0,1) \text { such that } \mathrm{w}=\mu \mathcal{M} \mathrm{w}\} .
$$

$\Theta$ is bounded in $\mathfrak{Z}$. Take $\mathrm{w} \in \Theta$ and $\mu \in[0,1)$ such that $\mathrm{w}=\mu \mathcal{M} \mathrm{w}$ then

$$
\begin{aligned}
\|\mathrm{w}\| & =\mu\|\mathcal{M} \mathrm{w}\| \leq \mu(\|\mathcal{A} \mathrm{w}\|+\|\mathcal{B} \mathrm{w}\|) \\
& \leq \mu\left[\mathfrak{a}^{\prime}\|\mathrm{w}\|^{\mathfrak{c}_{1}}+\mathfrak{b}^{\prime}+\mathfrak{c}^{\prime}\|\mathrm{w}\|^{\mathfrak{c}_{2}}+\mathfrak{d}^{\prime}\right] .
\end{aligned}
$$

Thus $\Theta$ is bounded in $\mathfrak{Z}$ for $\mathfrak{c}_{1}<1, \mathfrak{c}_{2}<1$. Therefore, Theorem 1 guarantees that $\mathcal{M}$ possesses at least one fixed point, and the set of the fixed points of $\mathcal{M}$ is bounded in $\mathfrak{Z}$. Hence the considered problem has at least one solution.

Let the given condition hold: 
(iv) There exist constants $\mathcal{L}_{\mathrm{t}}>0, \mathcal{L}_{\mathfrak{f}}>0$ such that

$$
\begin{aligned}
& \mid \mathfrak{f}\left(\mathrm{t}, \mathrm{u}(\mathrm{t}), \mathrm{u}\left(\gamma_{1} \mathrm{t}\right), \cdots, \mathrm{u}\left(\gamma_{\mathrm{n}} \mathrm{t}\right)\right)-\mathfrak{f}\left(\mathrm{t}, \mathrm{w}(\mathrm{t}), \mathrm{w}\left(\gamma_{1} \mathrm{t}\right), \cdots, \mathrm{w}\left(\gamma_{\mathrm{n}} \mathrm{t}\right) \mid\right. \\
& \leq \mathcal{L}_{\mathrm{t}}\left[|\mathrm{u}(\mathrm{t})-\mathrm{w}(\mathrm{t})|+\left|\mathrm{u}\left(\gamma_{1} \mathrm{t}\right)-\mathrm{w}\left(\gamma_{1} \mathrm{t}\right)\right|+\cdots+\left|\mathrm{u}\left(\gamma_{\mathrm{n}} \mathrm{t}\right)-\mathrm{w}\left(\gamma_{\mathrm{n}} \mathrm{t}\right)\right|\right] \\
& \leq \mathcal{L}_{\mathfrak{f}}|\mathrm{u}(\mathrm{t})-\mathrm{w}(\mathrm{t})|
\end{aligned}
$$

where $\mathcal{L}_{\mathfrak{f}}=(\mathrm{n}+1) \mathcal{L}_{\mathrm{t}}, \forall \mathrm{t} \in \mathrm{J}$, and $\mathrm{u}, \mathrm{w} \in \mathrm{R}$.

Theorem 3. Under hypotheses (i)-(iv), let $\exists$ a constant $\mathrm{r}>0$ such that

$$
\mathrm{r}=\left(\mathcal{K}_{1}+\mathcal{L}_{\mathfrak{f}}\right)\left(\frac{\theta^{\kappa}}{\Gamma(\kappa+1)}\right)<1,
$$

then (19) possesses at most one solution. Consequently our considered problem (5) has at most one solution.

Proof. Thanks to the Banach fixed point theorem for $\mathrm{u}, \mathrm{w} \in \mathfrak{Z}$, take

$$
\begin{aligned}
\|\mathcal{M u}-\mathcal{M} w\| & \leq\|\mathcal{A} \mathrm{u}-\mathcal{A} \mathrm{w}\|+\|\mathcal{B} \mathrm{u}-\mathcal{B} \mathrm{w}\| \\
& \leq\left(\mathcal{K}_{1}+\mathcal{L}_{\mathfrak{f}}\right)\left(\frac{\theta^{\kappa}}{\Gamma(\kappa+1)}\right)\|\mathrm{u}-\mathrm{w}\| \\
& =\mathrm{r}\|\mathrm{u}-\mathrm{w}\| .
\end{aligned}
$$

Hence, problem (19) possesses at most one solution. Consequently our considered problem (5) has at most one solution.

\section{Stability}

Now we provide stability results for the problem (5). Here we say that the goal of stability analysis of time delay problems/systems is to find the region in the delay parameter space where the considered problem/system is still stable. In fact, in dynamical problems, we search for a fixed point also called an equilibrium point and its stability. Therefore, investigating UH stability and its different kinds, we do not need an exact equilibrium point (exact solution) but there exists a close exact solution (fixed point) when the system is UH or UHR stable. First we provide a lemma which will help in establishing stability analysis.

Lemma 3. For the perturb problem

$$
\begin{aligned}
& { }^{\mathrm{c}} \mathrm{D}_{0+}^{\kappa} \mathrm{w}(\mathrm{t})=\mathfrak{f}\left(\mathrm{t}, \mathrm{w}(\mathrm{t}), \mathrm{w}\left(\gamma_{1} \mathrm{t}\right), \cdots, \mathrm{w}\left(\gamma_{\mathrm{n}} \mathrm{t}\right)\right)+\eta(\mathrm{t}), \forall \mathrm{t} \in \mathrm{J}, 0<\kappa \leq 1, \\
& \mathrm{w}(0)=\mathrm{w}_{0}+\int_{0}^{\theta} \frac{(\theta-\mathrm{s})^{\kappa-1}}{\Gamma(\kappa)} \mathfrak{g}(\mathrm{s}, \mathrm{w}) \mathrm{ds},
\end{aligned}
$$

the following holds

$$
|\mathrm{w}(\mathrm{t})-\mathcal{M} \mathrm{w}| \leq \frac{\theta^{\kappa} \epsilon}{\Gamma(\kappa+1)}, \quad \mathrm{t} \in \mathrm{J}
$$

Proof. By Lemma 2 the solution of perturb problem (21) is given by

$$
\mathrm{w}(\mathrm{t})=\mathcal{M} \mathrm{w}+\int_{0}^{\mathrm{t}} \frac{(\mathrm{t}-\mathrm{s})^{\kappa-1}}{\Gamma(\kappa)} \eta(\mathrm{s}) \mathrm{ds} .
$$


By Remark 1, we get

$$
\begin{aligned}
|\mathrm{w}(\mathrm{t})-\mathcal{M} \mathrm{w}| & \leq \int_{0}^{\mathrm{t}}\left|\frac{(\mathrm{t}-\mathrm{s})^{\kappa-1}}{\Gamma(\kappa)}\right| \eta(\mathrm{s}) \mid \mathrm{ds} \\
& \leq \frac{\theta^{\kappa} \epsilon}{\Gamma(\kappa+1)}
\end{aligned}
$$

Theorem 4. By assumption (iv) and Lemma 3, problem (5) is UH and GUH stable if $\Gamma(\kappa+1) \neq\left(\mathcal{K}_{1}+\right.$ $\left.\mathcal{L}_{\mathfrak{f}}\right) \theta^{\kappa}$ holds.

Proof. Let $\mathrm{w} \in \mathcal{Y}$ be at most one result of (5) and $\overline{\mathrm{w}}$ be any other solution of (21), then

$$
\begin{aligned}
\|\overline{\mathrm{w}}-\mathrm{w}\| & =\|\overline{\mathrm{w}}-\mathcal{M} \mathrm{w}\| \\
& \leq\|\overline{\mathrm{w}}-\mathcal{M} \overline{\mathrm{w}}\|+\|\mathcal{M} \overline{\mathrm{w}}-\mathcal{M} \mathrm{w}\| \\
& \leq \frac{\theta^{\kappa} \epsilon}{\Gamma(\kappa+1)}+\left(\mathcal{K}_{1}+\mathcal{L}_{\mathfrak{f}}\right)\left(\frac{\theta^{\kappa}}{\Gamma(\kappa+1)}\right)\|\overline{\mathrm{w}}-\mathrm{w}\| \\
& =\mathcal{C}_{\mathrm{q}} \epsilon, \quad \text { where } \quad \mathcal{C}_{\mathfrak{q}}=\frac{\theta^{\kappa}}{\Gamma(\kappa+1)-\left(\mathcal{K}_{1}+\mathcal{L}_{\mathfrak{f}}\right) \theta^{\kappa}} .
\end{aligned}
$$

Hence problem (5) is UH stable. For GUH stability if $\exists$ a nondecreasing function $\xi:(0,1) \rightarrow(0, \infty)$ such that $\xi(\epsilon)=\epsilon$ and $\xi(0)=0$, then the above inequality gives,

$$
\|\overline{\mathrm{w}}-\mathrm{w}\| \leq \mathcal{C}_{\mathrm{q}} \xi(\epsilon) .
$$

which shows GUH stability of (5).

Lemma 4. For perturb problem (21) the relation provided by

$$
|\mathrm{w}(\mathrm{t})-\mathcal{M w}| \leq \frac{\theta^{\kappa} \varphi \epsilon}{\Gamma(\kappa+1)}, \mathrm{t} \in \mathrm{J}
$$

holds.

Proof. Similarly we can prove Lemma 3.

Theorem 5. By assumption (iv) and Lemma 4 problem (5) is UHR and GUHR stable if $\Gamma(\kappa+1) \neq\left(\mathcal{K}_{1}+\right.$ $\left.\mathcal{L}_{\mathfrak{f}}\right) \theta^{\kappa}$ holds.

Proof. Let $\mathrm{w} \in \mathcal{Y}$ be a unique solution of (5) and $\overline{\mathrm{w}}$ be any solution of (21), then

$$
\begin{aligned}
\|\overline{\mathrm{w}}-\mathrm{w}\| & =\|\overline{\mathrm{w}}-\mathcal{M} \mathrm{w}\| \\
& \leq\|\overline{\mathrm{w}}-\mathcal{M} \overline{\mathrm{w}}\|+\|\mathcal{M} \overline{\mathrm{w}}-\mathcal{M} \mathrm{w}\| \\
& \leq \frac{\theta^{\kappa} \varphi \epsilon}{\Gamma(\kappa+1)}+\left(\mathcal{K}_{1}+\mathcal{L}_{\mathfrak{f}}\right)\left(\frac{\theta^{\kappa}}{\Gamma(\kappa+1)}\right)\|\overline{\mathrm{w}}-\mathrm{w}\| \\
& =\mathcal{C}_{\mathrm{q}, \varphi} \varphi(\mathrm{t}) \epsilon, \quad \text { where } \quad \mathcal{C}_{\mathrm{q}, \varphi}=\frac{\theta^{\kappa}}{\Gamma(\kappa+1)-\left(\mathcal{K}_{1}+\mathcal{L}_{\mathfrak{f}}\right) \theta^{\kappa}} .
\end{aligned}
$$

Hence the problem (5) is UHR stable. Now using the above inequality with

$$
\mathcal{C}_{\mathrm{q}, \varphi}=\frac{\theta^{\kappa} \epsilon}{\Gamma(\kappa+1)-\left(\mathcal{K}_{1}+\mathcal{L}_{\mathfrak{f}}\right) \theta^{\kappa}}
$$


we have

$$
\|\overline{\mathrm{w}}-\mathrm{w}\| \leq \mathcal{C}_{\mathrm{q}, \varphi} \varphi(\mathrm{t}) .
$$

Thus problem (5) is GUHR stable.

\section{Application of Aforesaid Analysis}

Here we present some applications for our analysis.

Example 1. Consider the given problem as

$$
\begin{aligned}
{ }^{c} \mathrm{D}_{0+}^{\frac{1}{2}} \mathrm{w}(\mathrm{t}) & =\mathrm{t}^{2}+\frac{1}{100}\left(\frac{|\mathrm{w}(\mathrm{t})|}{1+|\mathrm{w}(\mathrm{t})|^{\frac{1}{2}}}+\frac{\left|\mathrm{w}\left(\gamma_{1} \mathrm{t}\right)\right|}{1+\left|\mathrm{w}\left(\gamma_{1} \mathrm{t}\right)\right|^{\frac{1}{2}}}+\frac{\left|\mathrm{w}\left(\gamma_{2} \mathrm{t}\right)\right|}{1+\left|\mathrm{w}\left(\gamma_{2} \mathrm{t}\right)\right|^{\frac{1}{2}}}\right. \\
& \left.+\frac{\left|\mathrm{w}\left(\gamma_{3} \mathrm{t}\right)\right|}{1+\left|\mathrm{w}\left(\gamma_{3} \mathrm{t}\right)\right|^{\frac{1}{2}}}\right) \\
\mathrm{w}(0) & =\mathrm{w}_{0}+\int_{0}^{1} \frac{1}{500} \frac{(1-\mathrm{s})^{\frac{-1}{2}}}{\Gamma\left(\frac{1}{2}\right)}\left(\frac{\mathrm{w}(\mathrm{s})}{1+|\mathrm{w}(\mathrm{s})|^{\frac{1}{2}}}\right) \mathrm{ds} .
\end{aligned}
$$

Here we have

$$
\begin{aligned}
& \mathfrak{f}\left(\mathrm{t}, \mathrm{w}(\mathrm{t}), \mathrm{w}\left(\gamma_{1} \mathrm{t}\right), \mathrm{w}\left(\gamma_{2} \mathrm{t}\right), \mathrm{w}\left(\gamma_{3} \mathrm{t}\right)\right)=\mathrm{t}^{2}+\frac{1}{100}\left(\frac{|\mathrm{w}(\mathrm{t})|}{1+|\mathrm{w}(\mathrm{t})|^{\frac{1}{2}}}+\frac{\left|\mathrm{w}\left(\gamma_{1} \mathrm{t}\right)\right|}{1+\left|\mathrm{w}\left(\gamma_{1} \mathrm{t}\right)\right|^{\frac{1}{2}}}\right. \\
& \left.+\frac{\left|\mathrm{w}\left(\gamma_{2} \mathrm{t}\right)\right|}{1+\left|\mathrm{w}\left(\gamma_{2} \mathrm{t}\right)\right|^{\frac{1}{2}}}+\frac{\left|\mathrm{w}\left(\gamma_{3} \mathrm{t}\right)\right|}{1+\left|\mathrm{w}\left(\gamma_{3} \mathrm{t}\right)\right|^{\frac{1}{2}}}\right), \mathfrak{g}(\mathrm{t}, \mathrm{w})=\frac{1}{500}\left(\frac{\mathrm{w}(\mathrm{t})}{1+|\mathrm{w}(\mathrm{t})|^{\frac{1}{2}}}\right) .
\end{aligned}
$$

Now $\mathfrak{f}, \mathfrak{g}$ satisfies conditions ( (i)-(iii) for $\mathcal{\kappa}=\frac{1}{2}, \mathrm{~J}=[0,1], \mathfrak{a}=\mathcal{K}_{1}=\frac{1}{500}, \mathfrak{b}=0, \mathfrak{c}_{1}=\mathfrak{c}_{2}=\frac{1}{2}, \mathfrak{c}_{\mathfrak{f}}=\frac{1}{25}$, $\mathfrak{d}=1$ and $\mathrm{w}_{0}=1$, consider the set

$$
\Theta=\{\mathrm{w} \in \mathfrak{Z}: \exists \mu \in[0,1] \text { such that } \mathrm{w}=\mu \mathcal{M} \mathrm{w}\} .
$$

Let $\mathrm{w} \in \Theta$ and $\mu \in[0,1]$, such that $\mathrm{w}=\mu \mathcal{M} \mathrm{w}$, then

$$
\begin{aligned}
\|\mathrm{w}\| & =\mu\|\mathcal{M} \mathrm{w}\| \leq \mu(\|\mathcal{A} \mathrm{w}\|+\|\mathcal{B} \mathrm{w}\|) \\
& \leq \mu\left[\mathfrak{a}^{\prime}\|\mathrm{w}\|^{\mathfrak{c}_{1}}+\mathfrak{b}^{\prime}+\mathfrak{c}^{\prime}\|\mathrm{w}\|^{\mathfrak{c}_{2}}+\mathfrak{d}^{\prime}\right] \\
& \leq \mu\left[\frac{1}{250 \sqrt{\pi}}\|\mathrm{w}\|^{\frac{1}{2}}+1+\frac{2}{25 \sqrt{\pi}}\|\mathrm{w}\|^{\frac{1}{2}}+\frac{2}{\sqrt{\pi}}\right],
\end{aligned}
$$

which shows $\Theta$ is bounded in $\mathfrak{Z}$. Thus by using Theorem 2, the problem (22) possesses at least one solution and the set of solutions is bounded.

For uniqueness, if $\mathfrak{f}$ satisfies condition (iv) with $\mathcal{L}_{\mathfrak{f}}=\frac{1}{25}$, then

$$
\mathrm{r} \approx 0.0472<1 \text {. }
$$

Hence Theorem 3 guarantees that problem (22) has a unique solution.

The problem (22) is UR and GUH stable, since $\Gamma(\kappa+1) \neq\left(\mathcal{K}_{1}+\mathcal{L}_{\mathfrak{f}}\right) \theta^{\kappa}$ for $\mathcal{L}_{\mathfrak{f}}=\frac{1}{25}, \theta=1$, $\kappa=\frac{1}{2}, \mathcal{K}_{1}=\frac{1}{500}$. Similarly problem (22) is UHR and GUHR stable with $\varphi(\mathrm{t})=\mathrm{t}$ for $\mathrm{t} \in(0,1)$. 
Example 2. Now, we discuss the same analysis for the problem given below:

$$
\begin{aligned}
{ }^{c} \mathrm{D}_{0+}^{\frac{1}{5}} \mathrm{w}(\mathrm{t}) & =\frac{1}{\sqrt{\exp (\mathrm{t})}}+\frac{1}{5} \frac{|\mathrm{w}(\mathrm{t})|+\sin \left(\left|\mathrm{w}\left(\gamma_{1} \mathrm{t}\right)\right|\right)}{1+\left|\mathrm{w}\left(\gamma_{2} \mathrm{t}\right)\right|^{\frac{1}{4}}} \\
\mathrm{w}(0) & =\mathrm{w}_{0}+\int_{0}^{1} \frac{(1-\mathrm{s})^{\frac{-4}{5}}}{\Gamma\left(\frac{1}{5}\right)} \frac{1}{10}(\mathrm{~s}+\mathrm{w}(\mathrm{s})) \mathrm{ds} .
\end{aligned}
$$

Here we have

$$
\begin{aligned}
& \mathfrak{f}\left(\mathrm{t}, \mathrm{w}(\mathrm{t}), \mathrm{w}\left(\gamma_{1} \mathrm{t}\right), \mathrm{w}\left(\gamma_{2} \mathrm{t}\right)\right)=\frac{1}{\sqrt{\exp (t)}}+\frac{1}{5} \frac{|\mathrm{w}(\mathrm{t})|+\sin \left(\left|\mathrm{w}\left(\gamma_{1} \mathrm{t}\right)\right|\right)}{1+\left|\mathrm{w}\left(\gamma_{2} \mathrm{t}\right)\right|^{\frac{1}{4}}} \text { and } \\
& \mathfrak{g}(\mathrm{t}, \mathrm{w})=\frac{1}{10}(\mathrm{t}+\mathrm{w}(\mathrm{t})) .
\end{aligned}
$$

Now $\mathfrak{f}, \mathfrak{g}$ satisfies condition (i)_(iii) for $\kappa=\frac{1}{5}, J=[0,1], \mathfrak{a}=\mathfrak{b}=\mathcal{K}_{1}=\frac{1}{10}, \mathfrak{c}_{1}=1, \mathfrak{c}_{2}=\frac{3}{4}, \mathfrak{c}_{\mathfrak{f}}=\frac{2}{5}$, $\mathfrak{d}=\mathrm{w}_{0}=1$; consider the set

$$
\Theta=\{\mathrm{w} \in \mathfrak{Z}: \exists \mu \in[0,1] \text { such that } \mathrm{w}=\mu \mathcal{M} \mathrm{w}\} .
$$

Let $\mathrm{w} \in \Theta$ and $\mu \in[0,1]$, such that $\mathrm{w}=\mu \mathcal{M} \mathrm{w}$, then

$$
\begin{aligned}
\|\mathrm{w}\| & =\mu\|\mathcal{M} \mathrm{w}\| \leq \mu(\|\mathcal{A} \mathrm{w}\|+\|\mathcal{B} \mathrm{w}\|) \\
& \leq \mu\left[\mathfrak{a}^{\prime}\|\mathrm{w}\|^{\mathfrak{c}_{1}}+\mathfrak{b}^{\prime}+\mathfrak{c}^{\prime}\|\mathrm{w}\|^{\mathfrak{c}_{2}}+\mathfrak{d}^{\prime}\right] \\
& \leq \mu\left[\frac{1}{2 \Gamma\left(\frac{1}{5}\right)}\|\mathrm{w}\|+1+\frac{1}{2 \Gamma\left(\frac{1}{5}\right)}+\frac{2}{\Gamma\left(\frac{1}{5}\right)}\|\mathrm{w}\|^{\frac{3}{4}}+\frac{5}{\Gamma\left(\frac{1}{5}\right)}\right],
\end{aligned}
$$

which shows $\Theta$ is bonded in $\mathfrak{Z}$. Thus by using Theorem 2, problem (23) possesses at least one solution and the set of solutions is bounded.

For uniqueness, if $\mathfrak{f}$ satisfies condition (iv) with $\mathcal{L}_{\mathfrak{f}}=\frac{2}{5}$, then

$$
\mathrm{r} \approx 0.544563<1 .
$$

Hence Theorem 3 guarantees that problem (23) has a unique solution.

The problem (23) is UH and GUH stable, since $\Gamma(\kappa+1) \neq\left(\mathcal{K}_{1}+\mathcal{L}_{\mathfrak{f}}\right) \theta^{\kappa}$ for $\mathcal{L}_{\mathfrak{f}}=\frac{2}{5}, \theta=1$, $\kappa=\frac{1}{5}, \mathcal{K}_{1}=\frac{1}{10}$. Similarly problem (23) is UHR and GUHR stable with $\varphi(\mathrm{t})=\mathrm{t}$ for $\mathrm{t} \in(0,1)$.

Example 3. Let us take another problem as

$$
\begin{aligned}
{ }^{c} \mathrm{D}_{0+}^{\frac{7}{9}} \mathrm{w}(\mathrm{t}) & =10 \exp (-t)+\left(-\frac{1}{20}\left(\frac{|\mathrm{w}(\mathrm{t})|+\left|\mathrm{w}\left(\gamma_{1} \mathrm{t}\right)\right|+\left|\mathrm{w}\left(\gamma_{2} \mathrm{t}\right)\right|}{5 \exp (t)+\left|\mathrm{w}\left(\gamma_{3} \mathrm{t}\right)\right|^{\frac{1}{3}}}\right)\right) \\
\mathrm{w}(0) & =\int_{0}^{1} \frac{(1-\mathrm{s})^{\frac{-2}{9}}}{\Gamma\left(\frac{7}{9}\right)} \frac{1}{5}\left(\frac{\mathrm{w}(\mathrm{s})}{1+|\sin (\mathrm{w}(\mathrm{s}))|^{\frac{3}{5}}}\right) \mathrm{ds} .
\end{aligned}
$$

Here we have

$$
\begin{aligned}
& \mathfrak{f}\left(\mathrm{t}, \mathrm{w}(\mathrm{t}), \mathrm{w}\left(\gamma_{1} \mathrm{t}\right), \mathrm{w}\left(\gamma_{2} \mathrm{t}\right), \mathrm{w}\left(\gamma_{3} \mathrm{t}\right)\right)=10 \exp (-t)+\left(-\frac{1}{20}\left(\frac{|\mathrm{w}(\mathrm{t})|+\left|\mathrm{w}\left(\gamma_{1} \mathrm{t}\right)\right|+\left|\mathrm{w}\left(\gamma_{2} \mathrm{t}\right)\right|}{5 \exp (t)+\left|\mathrm{w}\left(\gamma_{3} \mathrm{t}\right)\right|^{\frac{1}{3}}}\right)\right) \mathrm{dt}, \\
& \mathfrak{g}(\mathrm{t}, \mathrm{w})=\frac{1}{5}\left(\frac{\mathrm{w}(\mathrm{t})}{1+|\sin (\mathrm{w}(\mathrm{t}))|^{\frac{3}{5}}}\right) .
\end{aligned}
$$


Now $\mathfrak{f}, \mathfrak{g}$ satisfies conditions (i)-(iii) for $\mathcal{\kappa}=\frac{7}{9}, \mathrm{~J}=[0,1], \mathfrak{a}=\mathcal{K}_{1}=\frac{1}{5}, \mathfrak{b}=0, \mathfrak{c}_{1}=\frac{2}{5}, \mathfrak{c}_{2}=\frac{2}{3}, \mathfrak{c}_{\mathfrak{f}}=\frac{3}{20}$, $\mathfrak{d}=10$ and $\mathrm{w}_{0}=0$, consider the set

$$
\Theta=\{\mathrm{w} \in \mathfrak{Z}: \exists \mu \in[0,1] \text { such that } \mathrm{w}=\mu \mathcal{M} \mathrm{w}\} \text {. }
$$

Let $\mathrm{w} \in \Theta$ and $\mu \in[0,1]$, such that $\mathrm{w}=\mu \mathcal{M} \mathrm{w}$, then

$$
\begin{aligned}
\|\mathrm{w}\| & =\mu\|\mathcal{M} \mathrm{w}\| \leq \mu(\|\mathcal{A} \mathrm{w}\|+\|\mathcal{B} \mathrm{w}\|) \\
& \leq \mu\left[\mathfrak{a}^{\prime}\|\mathrm{w}\|^{\mathfrak{c}_{1}}+\mathfrak{b}^{\prime}+\mathfrak{c}^{\prime}\|\mathrm{w}\|^{\mathfrak{c}_{2}}+\mathfrak{d}^{\prime}\right] \\
& \leq \mu\left[\frac{9}{35 \Gamma\left(\frac{7}{9}\right)}\|\mathrm{w}\|^{\frac{2}{5}}+\frac{27}{140 \Gamma\left(\frac{7}{9}\right)}\|\mathrm{w}\|^{\frac{2}{3}}+\frac{10}{7 \Gamma\left(\frac{7}{9}\right)}\right],
\end{aligned}
$$

which shows $\Theta$ is bonded in $\mathfrak{Z}$. Thus by using Theorem 2, the problem (24) possesses at least one solution and the set of solutions is bounded.

For uniqueness, if $\mathfrak{f}$ satisfies condition (iv) with $\mathcal{L}_{\mathfrak{f}}=\frac{3}{20}$, then

$$
\mathrm{r} \approx 0.378103<1 \text {. }
$$

Hence Theorem 3 guarantees that problem (24) has a unique solution.

The problem (24) is UH and GUH stable, since $\Gamma(\kappa+1) \neq\left(\mathcal{K}_{1}+\mathcal{L}_{\mathfrak{f}}\right) \theta^{\kappa}$ for $\mathcal{L}_{\mathfrak{f}}=\frac{3}{20}, \theta=1, \kappa=$ $\overline{9}, \mathcal{K}_{1}=\frac{1}{5}$. Similarly problem (24) is UHR and GUHR stable with $\varphi(\mathrm{t})=\mathrm{t}$ for $\mathrm{t} \in(0,1)$.

\section{Concluding Remarks}

Upon the application of the tool of nonlinear analysis known as the degree theory, we have successfully established some sufficient results for the existence and uniqueness of the solution to multi-terms delay FODEs under nonlinear integral boundary conditions. Further, some stable results regarding Ulam and its various kinds have also been derived via nonlinear functional analysis. The whole theoretical results have been testified to by providing some interesting examples. Hence, we claim that the suggested method can be used as a strong technique instead of the usual fixed point theory to study nonlinear delay FODEs under different boundary conditions. In future, we will extend the topological degree theory approach to more general problems involving other kinds of fractional derivatives like Riemann-Liouvile, Hadamard and Caputo-Fabrizo.

Author Contributions: All authors have contributed equally to this paper. All authors have read and agreed to the published version of the manuscript.

Funding: This research work was supported by HED of KPK Government under grant No: HEREF-46 and HEC of Pakistan under grant No: 10039, and by the Slovak Research and Development Agency under the contract No. APVV-18-0308 and by the Slovak Grant Agency VEGA No. 1/0358/20 and No. 2/0127/20.

Acknowledgments: We are thankful to the reviewers for their useful and constructive suggestions which improved this paper very well.

Conflicts of Interest: The authors declare no conflict of interest.

\section{References}

1. Liu, F.; Burrage, K. Novel techniques in parameter estimation for fractional dynamical models arising from biological systems. Comput. Math. Appl. 2011, 62, 822-833. [CrossRef]

2. Hilfer, R. Applications of Fractional Calculus in Physics; World Scientific: Singapore, 2000.

3. Lakshmikantham, V.; Leela, S.; Devi, J.V. Theory of Fractional Dynamic Systems; Cambridge Academic Publishers: Cambridge, UK, 2009.

4. Longhi, S. Fractional Schrödinger equation in optics. Opt. Lett. 2015, 40, 1117-1120. [CrossRef] [PubMed]

5. Liemert, A.; Kienle, A. Fractional Schrödinger equation in the presence of the linear potential. Mathematics 2016, 4, 31-45. [CrossRef] 
6. El-Nabulsi, R.A. Fractional derivatives generalization of Einstein's field equations. Indian J. Phys. 2013, 87, 195-200. [CrossRef]

7. Wang, X.; Wang, L.; Zeng, Q. Fractional differential equations with integral boundary conditions. J. Nonlinear Sci. Appl. 2015, 8, 309-314. [CrossRef]

8. Balachandran, K.; Kiruthika, S.; Trujillo, J.J. Existence results for fractional impulsive integrodifferential equations in Banach spaces. Commun. Nonlinear Sci. Numer. Simul. 2011, 16, 1970-1977. [CrossRef]

9. Rehman, M.; Khan, R.A. Existence and uniqueness of solutions for multi-point boundary value problems for fractional differential equations. Appl. Math. Lett. 2010, 23, 1038-1044. [CrossRef]

10. Kilbas, A.A.; Srivastava, H.M.; Trujillo, J.J. Theory and Applications of Fractional Differential Equations (North-Holland Mathematics Studies); Elsevier Science: Amsterdam, The Netherlands, 2006; Volume 204.

11. Mawhin, J. Topological Degree Methods in Nonlinear Boundary Value Problems. In CMBS Regional Conference Series in Mathematics; American Mathematical Society: Providence, RI, USA, 1979; Volume 40.

12. Isaia, F. On a nonlinear integral equation without compactness. Acta Math. Univ. Comen. 2006, 75, $233-240$.

13. Ockendon, J.; Tayler, A.B. The dynamics of a current collection system for an electric locomotive. Proc. R. Soc. Lond. A Math. Phys. Eng. Sci. 1971, 322, 447-468.

14. Balachandran, K.; Kiruthika, S. Fractional pantograph equation. Acta Math. Sci. 2013, 33, 712-720. [CrossRef]

15. Sedaghat, S.; Ordokhani, Y.; Dehghan, M. Numerical solution of the delay differential equations of pantograph type via Chebyshev polynomials. Commun. Nonlinear Sci. Numer. Simul. 2012, 17, 4815-4830. [CrossRef]

16. Liu, M.Z.; Li, D. Properties of analytic solution and numerical solution of multi-pantograph equation. Appl. Math. Comput. 2004, 155, 853-871. [CrossRef]

17. Sabri, A. An analysis of exponential stability of delayed neural networks with time varying delays. Neural Netw. 2004, 17, 1027-1031.

18. Jamal, D.; Riedinger, P.; Iung, C. Stability analysis and control synthesis for switched systems: A switched Lyapunov function approach. IEEE Trans. Autom. Control 2002, 47, 1883-1887.

19. Ullam, S.M. Problems in Modern Mathematics (Chapter VI); Wiley: New York, NY, USA, 1940.

20. Hyers, D.H. On the stability of the linear functional equation. Proc. Natl. Acad. Sci. USA 2015, 27, $222-224$. [CrossRef] [PubMed]

21. Wang, J.; Fečkan, M.; Zhou, Y. Ullam stability of impulsive ordinary differential equations. J. Math. Anal. Appl. 2012, 395, 258-264. [CrossRef]

22. Rus, I.A. Ulam stabilities of ordinary differential equations in a Banach space. Carpathian J. Math. 2010, 26, 103-107.

23. Podlubny, I. Fractional Differential Equations; Mathematics in Science and Engineering; Academic Press: New York, NY, USA, 1999.

24. Ntouyas, S.K. Nonlocal initial and boundary value problems: A survey. In Handbook of Differential Equations: Ordinary Differential Equations; Elsevier: Amsterdam, The Netherlands, 2005; Volume 2, pp. 461-557.

25. Webb, J.R.L.; Infante, G. Positive solutions of nonlocal boundary value problems involving integral conditions. Nonlinear Differ. Equ. Appl. 2008, 15, 45-67. [CrossRef]

26. Wang, J.R.; Zhou, Y.; Fečkan, M. Nonlinear impulsive problems for fractional differential equations and Ulam stability. Comput. Math. Appl. 2012, 64, 3389-3405. [CrossRef]

(C) 2020 by the authors. Licensee MDPI, Basel, Switzerland. This article is an open access article distributed under the terms and conditions of the Creative Commons Attribution (CC BY) license (http://creativecommons.org/licenses/by/4.0/). 